\title{
Immunomodulatory Effect of Hypertonic Saline Solution in Traumatic Brain-Injured Patients and Intracranial Hypertension
}

\author{
Luis R. Moscote-Salazar ${ }^{4} \quad$ Amit Agrawal ${ }^{2}$ \\ ${ }^{1}$ Faculty of Medicine, Universidad El Bosque, Bogotá, Colombia \\ ${ }^{2}$ Department of Neurosurgery, All India Institute of Medical \\ Sciences, Bhopal, Madhya Pradesh, India \\ ${ }^{3}$ Department of Medicine, Universidad Surcolombiana, \\ Huila, Colombia \\ ${ }^{4}$ Department of Neurocritical Care, Faculty of Medicine, \\ University of Cartagena, Cartagena, Colombia
}

Gabriel A. Quiñones-Ossa ${ }^{1}$ Adesh Shrivastava ${ }^{2}$ William Andres Florez Perdomo 3

\begin{abstract}
Address for correspondence Adesh Shrivastava, MBBS, MS, MCh, Department of Neurosurgery, All India Institute of Medical Sciences, Saket Nagar, Bhopal 462020, Madhya Pradesh, India (e-mail: dr.adesh.shrivastava@gmail.com).
\end{abstract}

Indian J Neurotrauma:2020;17:74-78

\begin{abstract}
Traumatic brain injury (TBI) is often associated with an increase in the intracranial pressure (ICP). This increase in ICP can cross the physiological range and lead to a reduction in cerebral perfusion pressure (CPP) and the resultant cerebral blood flow (CBF). It is this reduction in the CBF that leads to the secondary damage to the neural parenchyma along with the physical axonal and neuronal damage caused by the mass effect. In certain cases, a surgical intervention may be required to either remove the mass lesion (hematoma of contusion evacuation) or provide more space to the insulted brain to expand (decompressive craniectomy). Whether or not a surgical intervention

Keywords

- saline hypertonic solutions

- traumatic brain injury

- immunomodulation

- lymphocyte

- neutrophils

- intracranial pressure

- intracranial pressure increase

- immune system is performed, all these patients require some form of pharmaceutical antiedema agents to bring down the raised ICP. These agents have been broadly classified as colloids (e.g., mannitol, glycerol, urea) and crystalloids (e.g., hypertonic saline), and have been used since decades. Even though mannitol has been the workhorse for ICP reduction owing to its unique properties, crystalloids have been found to be the preferred agents, especially when long-term use is warranted. The safest and most widely used agent is hypertonic saline in various concentrations. Whatever be the concentration, hypertonic saline has created special interest among physicians owing to its additional property of immunomodulation and neuroprotection. In this review, we summarize and understand the various mechanism by which hypertonic saline exerts its immunomodulatory effects that helps in neuroprotection after TBI.
\end{abstract}

\section{Introduction}

Both colloids and crystalloids have been extensively used in the management of raised intracranial pressure (ICP) after traumatic brain injuries (TBIs). Compared with the colloid solutions, crystalloids better mimic the plasma characteristics and are associated with reduced incidences of alterations in electrolyte and $\mathrm{pH}$ balances. ${ }^{1,2}$ Crystalloid solutions have always been the preferred first-line agent in the fluid resuscitation for shock patients. ${ }^{3-11}$ They have recently become the preferred agents for osmotherapy for neurotrauma patients ${ }^{12,13}$ due to their ability to modulate the inflammatory reaction in the neural parenchyma. ${ }^{14-16}$ Numerous studies to establish the superiority of one over the other have been conducted in the past comparing mannitol and hypertonic saline. ${ }^{17-21}$ Even though no consensus could be reached,
DOI https://doi.org/

10.1055/s-0040-1713329

ISSN 0973-0508.
License terms

(ㅇ)( $\Theta \circledast$ 
individual recommendations have been made. ${ }^{17}$ Here we attempt to review the role of hypertonic saline in the management of TBI.

\section{Raised Intracranial Pressure}

The Monro-Kellie doctrine implies the need for management of raised ICP in patients with TBI to avoid secondary damage. In these cases, the measurement of ICP becomes an important outcome predictor. ${ }^{20}$ This ICP reading, along with the blood pressure, volume, and viscosity, determines the cerebral blood flow (CBF). This is the reason why reducing the ICP is the main objective in the management of TBI patients, ${ }^{11,21-25}$ and osmotherapy plays the central role in achieving this goal..$^{23-25}$

After the primary insult to the neuroparenchyma, if ICP rises, it leads to a fall in the cerebral perfusion pressure (CPP), which further leads to a reduction in CBF. This reduction in $\mathrm{CBF}$ is the major cause of secondary brain injury. ${ }^{26,27}$ Other than this, the next major cause of morbidity and mortality is acute blood loss (hemorrhagic shock), which may lead to massive tissue ischemia, an exaggerated systemic inflammatory response syndrome and multiorgan dysfunction syndrome (MODS). ${ }^{28}$ When this cascade sets in, it can be associated with cerebral death, neurologic deficits, permanent disability, brain herniation, and even death. ${ }^{9,21,23,29}$

The main objective of the management of TBIs is to control the ICP to maintain CPP and CBF in physiological ranges. ${ }^{26}$ Even before a surgical intervention is undertaken, osmotherapy is the first-line treatment in the medical management to reduce the raised ICP. ${ }^{30}$ The timing of initiation of hyperosmolar therapy is critical to contain the progressive neuronal loss after the trauma. ${ }^{10}$

\section{Intracranial Pressure and Physiological Effects of Hypertonic Saline Solution}

Intravenous fluid therapy with hypertonic saline solution (HSS) for reducing elevated ICP dates back to almost 100 years ago., 32,24,31 The physiological effects of the HSS are diverse, and there are multiple mechanisms through which it exerts its therapeutic effects. The basic mechanism of action of any hyperosmolar agent is to generate an osmotic gradient across the endothelial barrier between the endovascular and tissue environments. ${ }^{3}$ The HSS is a solution with a concentration of $\mathrm{NaCl}$ (sodium chloride) higher than the normal or physiological value. It has been extensively subjected to basic and clinical research in the last couple of decades. Its efficacy has been demonstrated in reducing an elevated ICP after TBI or due to other causes including intracranial bleeding of spontaneous or traumatic etiology. ${ }^{9} 18-20,22,23,29,32-36$ One of the main advantages of the HSS usage is the rapid reduction of the ICP with extended responsiveness greater than 2 hours without pressure rebound, which is related to improved neurologic outcomes. ${ }^{23}$

Additionally, HSS has shown other rheological effects such as plasma expansion, improvement in microcirculatory blood flow, reperfusion injury protection, and recovery of CPP, among others. ${ }^{8,10,11,29,37}$

Saline solution of $0.9 \%$ is used as standard replacement and maintenance fluid in neurosurgical patients. It is the preferred agent due to its very low propensity to cause intravenous fluid related cerebral edema. ${ }^{1,27}$ When used in hyperosmolar concentrations ( $3 \%$ or more), it exerts its ICP lowering effect, mostly caused by an osmotic gradient that produces a shift of free water from the interstitial and intracellular spaces to the intravascular space. ${ }^{11,12,22,33}$ This reduction in tissue fluid by the immediate hyperosmolar effect leads to a reduction in ICP. ${ }^{11,13,23}$ Bolus treatment with HSS is indicated in patients with refractory raised ICP, where intravascular fluid expansion helps maintaining CPP while reducing edema. ${ }^{20,22,23,29,33}$ The blood-brain barrier $(\mathrm{BBB})$ is a water permeable structure, which means that hypotonic solutions lead to a water shift to the brain while hypertonic solutions lead to brain dehydration. ${ }^{27} \mathrm{An}$ intact BBB is needed to achieve the beneficial effects of hyperosmolar therapy..$^{13}$ Consequently, on administration of any hyperosmolar agent, ICP reduction occurs in the uninjured brain regions where the BBB is intact. ${ }^{22,30}$ As opposed to this, in regions where BBB is damaged, there is a leakage of osmotic substances (proteins and electrolytes) to the brain parenchyma, causing an osmotic effect that leads to brain edema and subsequent adverse effects. ${ }^{12,13,22}$ The use of crystalloids (e.g., HSS) in these circumstances as against the use of colloids (e.g., mannitol) is thus preferable as $\mathrm{NaCl}$ being a normal constituent of the intracellular and extracellular spaces redistributes easily. This redistribution helps in avoiding rebound edema caused by the use of colloids. In addition to this, it is also observed that unlike colloids, prolonged use of HSS does not open up the endothelial tight junctions. In fact, it helps in reversing the endothelial cell swelling caused by inflammation and ion exchange disturbance due to the loss of adenosine triphosphate (ATP). ${ }^{22}$ This increases the capillary lumen, which in addition to reduced blood viscosity and improved CPP promotes better tissue perfusion. ${ }^{11}$ By this, it counteracts the cerebral vasospasm associated with subarachnoid hemorrhage in TBI patients, thereby improving the microcirculation. All these mechanisms have been found to reduce the overall rate of fatal outcomes. ${ }^{11}$ In fact, in severe TBI patients, serum biomarkers elevation could be useful in the prediction and prognosis of clinical complications and neurologic deficits and outcomes; ${ }^{38,39}$ the usage of HSS has been related to a significant reduction in the measurement of these biomarkers. Considering this, it can be concluded that HSS usage is associated with mitigation in the brain damage and improvement in the neurologic outcomes. ${ }^{11,39}$

\section{Immunomodulatory Effect of Hypertonic Saline Solution}

The immunomodulatory effects of HSS have been correlated to a multitude of factors in regulating the immune system. Laboratory evidence conclusively proves that HSS treatment affects the vasomotor tone by the release of nitric oxide, endothelins, and eicosanoids, as well as reversal in the macrophage proinflammatory effect to an anti-inflammatory one. ${ }^{11}$ Some studies have demonstrated that these effects of hypertonicity on the immune system are differentially mediated through the changes in lymphocyte and neutrophil functions.,31 
HSS effect is also related to the suppression of some functions such as expression of adhesion molecules, cytokines production, production of reactive oxygen species (ROS), phagocytic abilities, and degranulation. ${ }^{10}$ The understanding of these pathophysiological and immunomodulatory effects helps the physician in deciding the type of hyperosmolar therapy according to the patient's status and requirement.

\section{Effects of Hypertonic Saline on Neutrophil Functions}

The polymorphonuclear cells (PMN) are the first line of defense due to their ready stock of multiple enzymes within intracellular granules and are thus the first responders in any inflammatory condition. They are recruited at the site of inflammation within minutes of insult. Their rapid degranulation is considered as a trigger for the organ damage that occurs in shock patients. ${ }^{8,15}$ The rapidly progressive inflammatory cascade by neutrophil migration and degranulation is also responsible for organ failure in infectious states such as sepsis. ${ }^{22}$

PMN activation in trauma cases can lead to unnecessary tissue damage and severe posttraumatic complications in various organs including the brain. ${ }^{15,40}$ In these cases, it is the immunomodulatory effect of HSS that causes a reduction in neutrophil recruitment and activation, which might improve patient outcomes and prognosis. ${ }^{41}$ HSS resuscitation therapy has been found to reduce the priming and activation of the PMN by activating intracellular signaling cascades related to the cAMP (cyclic adenosine monophosphate) mediated pathways that suppress the cell activation processes.$^{28}$ Neutrophils suppression by the HSS also happens by means of reduction in the expression of adhesion molecule and production of ROS, as well as attenuation of the oxidative stress. . $^{1128,42-44}$

Another purported mechanism of action of HSS for reducing trauma-related inflammation is through the toll-like receptors (TLRs). PMNs along with the TLRs are essential compounds of the innate immunity. TLRs 1, 2, 4, 5, and 6 are expressed on the cell surface and recognize the bacterial products. TLRs 3, 7, 8, and 9 are expressed in the intracellular compartment and have an important role in the recognition of nucleic acids in the viral process. ${ }^{8,45}$ The activation of the TLRs regulates the chemokine receptors as well as stimulates A2a receptors that are linked to cAMP signaling cascade. And by this, both pathways regulate neutrophil functions, ${ }^{8,41}$ and HSS modifies the TLR-4 pathway, leading to the immunomodulation of the effects mediated by PMN cells. ${ }^{8}$

In vitro studies indicate that shock-mediated MODS is caused by the liberation of ROS, proteases, hydrolytic enzymes, and inflammatory cytokines, as well as by the neutrophil sequestration in vital organs. The usage of HSS is said to mitigate this pathway of damage as well. ${ }^{28}$

Similar to HSS, a combination of HSS with dextran (HSD) is also seen to attenuate the inflammatory response., ${ }^{3,46}$ A small volume of HSD for resuscitation has been found to abolish the CD11b upregulation, which happens in shock. This further leads to the reduction in the CD14 population and the subsequent proinflammatory subsets and tumor necrosis factor- $\alpha$ activity along with a concomitant increase in anti-inflammatory substances (interleukin [IL] 1ra and IL-10)., ${ }^{3,7}$ The usage of HSD is thus associated with less inflammatory and coagulation cascade activation that mitigates the secondary injury in TBI patients. ${ }^{23}$ But some researchers have concluded that HSD might exacerbate subclinical inflammatory response and, by this, be less effective than HSS in the mitigation of inflammatory status. ${ }^{28}$

In conclusion, HSS weakens the neutrophil activation and cytokines production, ${ }^{7,22}$ thus moderating the PMN cytotoxicity and dampening the inflammatory response..$^{40}$ The hypertonic environment reduces cellular edema that mitigates the cellular migration, ${ }^{11}$ decreases the rolling and adherence of PMNs to endothelial cells, and improves the microcirculation by negating the endothelial upregulation of adhesion molecules ( 1 and b2 integrins). ${ }^{10}$ However, there is a theoretical possibility that mitigation of the PMN might lead to unfavorable outcomes due to the increased risk of nosocomial infections by the inhibition of the "first line of defense" of the patient. ${ }^{11,41}$ Whether it is clinically relevant is still unknown.

\section{Hypertonic Saline Solutions and Lymphocyte Cells Functions}

Lymphocyte cells (B or T cells) are also a part of the immune system that takes over as the acute phase changes to subacute and chronic phases. Studies show that $\mathrm{T}$ cells are suppressed after an impairment of the cellular immune defense in cases of severe trauma. ${ }^{16}$ Administration of HSS is said to restore lymphocyte dysfunction. ${ }^{14,42}$ Though the mechanisms may not be very clear, but experimental data in intestinal mucosa have shown that HSS reduces the rate of apoptosis of lymphocytes after issue insult, thereby downregulating inflammation and improving the immune responses., ${ }^{3,5}$ The proposed mechanism may increase the IL-2 expression by releasing cellular ATP that activates and enhances the T-cell function. ${ }^{8}$ There are certain molecules that are related to ATP release, for example, pannexin-1 (PANX1). It is one of the three isoforms of the pannexin and is related to gap junctions proteins that mediate the ATP release from the T cells. ${ }^{16,41,46}$ Along with CBX-sensitive gap junctions, these channels commit to the ATP. ${ }^{41}$ In TBI patients, HSS may exert its immunomodulatory effect by controlling ATP release through the PANX1 hemichannels and stimulating the P2X1, P2X4, and P2X7 receptors that will promote a p38 MAPK activation with a gene transcription of IL-2. ${ }^{16}$

\section{Conclusions}

In the brain-injured patients, fluid therapy is used with the aim to maintain an optimal CBF and oxygenation. ${ }^{27}$ It is also known that patients who required neurosurgical procedure are susceptible to a sodium hemostasis disbalance. ${ }^{47}$ For the purpose of resuscitation, hypotonic solutions should not be used due to the risk of further hyponatremia and subsequent cerebral edema. ${ }^{1}$

Considering the previously mentioned, the use of HSS in the TBI context would help in the modulation of the inflammatory response and reduce the risk of brain swelling or edema that could lead to high ICP, and even lead to fatal outcomes. 


\section{Conflict of Interest}

None declared.

\section{References}

1 Lima MF, Neville IS, Cavalheiro S, Bourguignon DC, Pelosi P, Malbouisson LMS. Balanced crystalloids versus saline for perioperative intravenous fluid administration in children undergoing neurosurgery: a randomized clinical trial. J Neurosurg Anesthesiol 2019;31(1):30-35

2 Cecconi M, Hofer C, Teboul JL, et al; FENICE Investigators; ESICM Trial Group. Fluid challenges in intensive care: the FENICE study: a global inception cohort study. Intensive Care Med 2015;41(9):1529-1537

3 Silva MR e. Figueiredo LP de. Hypertonic saline for treatment of shock: have we looked for everything? Med Express 2014;1(1):14-21

4 de Felippe J Jr, Timoner J, Velasco IT, Lopes OU, Rocha-e-Silva M Jr. Treatment of refractory hypovolaemic shock by $7.5 \%$ sodium chloride injections. Lancet 1980;2(8202):1002-1004

5 Lu YQ Huang WD, Cai XJ, Gu LH, Mou HZ. Hypertonic saline resuscitation reduces apoptosis of intestinal mucosa in a rat model of hemorrhagic shock. J Zhejiang Univ Sci B 2008;9(11):879-884

6 Deree J, Martins JO, Leedom A, et al. Hypertonic saline and pentoxifylline reduces hemorrhagic shock resuscitationinduced pulmonary inflammation through attenuation of neutrophil degranulation and proinflammatory mediator synthesis. J Trauma 2007;62(1):104-111

7 Rizoli SB, Rhind SG, Shek PN, et al. The immunomodulatory effects of hypertonic saline resuscitation in patients sustaining traumatic hemorrhagic shock: a randomized, controlled, double-blinded trial. Ann Surg 2006;243(1):47-57

8 Choi SH, Yoon YH, Kim JY, Moon SW, Cho YD, Yeom JW. The effect of hypertonic saline on mRNA of proinflammatory cytokines in lipopolysaccharide-stimulated polymorphonuclear cells. Curr Ther Res Clin Exp 2014;76:58-62

9 Berger-Pelleiter E, Émond M, Lauzier F, Shields JF, Turgeon AF. Hypertonic saline in severe traumatic brain injury: a systematic review and meta-analysis of randomized controlled trials. CJEM 2016;18(2):112-120

10 Motaharinia J, Etezadi F, Moghaddas A, Mojtahedzadeh M. Immunomodulatory effect of hypertonic saline in hemorrhagic shock. Daru 2015;23(1):47

11 Bulger EM, Hoyt DB. Hypertonic resuscitation after severe injury: is it of benefit? Adv Surg 2012;46:73-85

12 White $\mathrm{H}$, Cook D, Venkatesh B, The use of hypertonic saline for treating intracranial hypertension after traumatic brain injury. Anesth Analg 2006;102(6):1836-1846

13 Ropper AH. Hyperosmolar therapy for raised intracranial pressure. N Engl J Med 2012;367(8):746-752

14 Kramer GC. Hypertonic resuscitation: physiologic mechanisms and recommendations for trauma care. J Trauma 2003;54(5, Suppl):S89-S99

15 Chen Y, Hashiguchi N, Yip L, Junger WG. Hypertonic saline enhances neutrophil elastase release through activation of P2 and A3 receptors. Am J Physiol Cell Physiol 2006;290(4): C1051-C1059

16 Woehrle T, Yip L, Manohar M, et al. Hypertonic stress regulates $\mathrm{T}$ cell function via pannexin-1 hemichannels and P2X receptors. J Leukoc Biol 2010;88(6):1181-1189

17 Carney N, Totten AM, O'Reilly C, et al. Guidelines for the management of severe traumatic brain injury, fourth editionNeurosurgery 2017;80(1):6-5

18 Dunham CM, Malik RJ, Huang GS, Kohli CM, Brocker BP, Ugokwe KT. Hypertonic saline administration and complex traumatic brain injury outcomes: a retrospective study. Int J Burns Trauma 2018;8(3):40-53

19 Mangat HS, Hypertonic saline infusion for treating intracranial hypertension after severe traumatic brain injury. Crit Care 2018;22(1):37

$20 \mathrm{Gu}$ J, Huang H, Huang Y, Sun H, Xu H. Hypertonic saline or mannitol for treating elevated intracranial pressure in traumatic brain injury: a meta-analysis of randomized controlled trials. Neurosurg Rev 2019;42(2):499-509

21 Colton K, Yang S, Hu PF, et al. Responsiveness to therapy for increased intracranial pressure in traumatic brain injury is associated with neurological outcome. Injury 2014;45(12):2084-2088

22 Strandvik GF. Hypertonic saline in critical care: a review of the literature and guidelines for use in hypotensive states and raised intracranial pressure. Anaesthesia 2009;64(9):990-1003

23 Alnemari AM, Krafcik BM, Mansour TR, Gaudin D, A comparison of pharmacologic therapeutic agents used for the reduction of intracranial pressure after traumatic brain injury. World Neurosurg 2017;106:509-528

24 Patil H, Gupta R. A comparative study of bolus dose of hypertonic saline, mannitol, and mannitol plus glycerol combination in patients with severe traumatic brain injury. World Neurosurg 2019;125:e221-e228

25 Shein SL, Ferguson NM, Kochanek PM, et al. Effectiveness of pharmacological therapies for intracranial hypertension in children with severe traumatic brain injury-results from an automated data collection system time-synched to drug administration. Pediatr Crit Care Med 2016;17(3):236-245

26 Scalfani MT, Dhar R, Zazulia AR, Videen TO, Diringer MN. Effect of osmotic agents on regional cerebral blood flow in traumatic brain injury. J Crit Care 2012;27(5):526.e7-526.e12

27 van der Jagt M, Fluid management of the neurological patient: a concise review. Crit Care 2016;20(1):126

28 Junger WG, Rhind SG, Rizoli SB, et al. Resuscitation of traumatic hemorrhagic shock patients with hypertonic saline-without dextran-inhibits neutrophil and endothelial cell activation. Shock 2012;38(4):341-350

29 Dias C, Silva MJ, Pereira E, et al. Post-traumatic multimodal brain monitoring: response to hypertonic saline. J Neurotrauma 2014;31(22):1872-1880

30 Geeraerts T, Velly L, Abdennour L, et al; French Society of Anaesthesia; Intensive Care Medicine; in partnership with Association de neuro-anesthésie-réanimation de langue française (Anarlf); French Society of Emergency Medicine (Société Française de Médecine d'urgence (SFMU); Société française de neurochirurgie (SFN); Groupe francophone de réanimation et d'urgences pédiatriques (GFRUP); Association des anesthésistes-réanimateurs pédiatriques d'expression française (Adarpef). Management of severe traumatic brain injury (first 24hours) Anaesth Crit Care Pain Med 2018;37(2): 171-186

31 Weed LH, McKibben PS. Pressure changes in the cerebro-spinal fluid following intravenous injection of solutions of various concentrations. Am J Physiol Content 1919;48(4):512-530

32 Jha RM, Kochanek PM. A precision medicine approach to cerebral edema and intracranial hypertension after severe traumatic brain injury: quo vadis? Curr Med Group 2018; 7;18(12):105

33 Pasarikovski CR, Alotaibi NM, Al-Mufti F, Macdonald RL, Hypertonic saline for increased intracranial pressure after aneurysmal subarachnoid hemorrhage: a systematic review. World Neurosurg 2017;105:1-6

34 Mangat HS, Wu X, Gerber LM, et al. Hypertonic saline is superior to mannitol for the combined effect on intracranial pressure and cerebral perfusion pressure burdens in patients with severe traumatic brain injury. Neurosurgery 2020;86(2):221-230 
35 Smith M. Refractory intracranial hypertension: the role of decompressive craniectomy. Anesth Analg 2017;125(6): 1999-2008

36 Abraham P, Rennert RC, Gabel BC, et al. ICP management in patients suffering from traumatic brain injury: a systematic review of randomized controlled trials. Acta Neurochir (Wien) 2017;159(12):2279-2287

37 Mortazavi MM, Romeo AK, Deep A, et al. Hypertonic saline for treating raised intracranial pressure: literature review with meta-analysis. J Neurosurg 2012;116(1):210-221

38 Quinones-Ossa G, Padilla-Zambrano H, Pal R, et al. Biomarkers in acute brain trauma: a narrative review. J Acute Dis 2019;8(1):1

39 Baker AJ, Rhind SG, Morrison LJ, et al. Resuscitation with hypertonic saline-dextran reduces serum biomarker levels and correlates with outcome in severe traumatic brain injury patients. J Neurotrauma 2009;26(8):1227-1240

40 Shields CJ, O'Sullivan AW, Wang JH, Winter DC, Kirwan WO, Redmond HP. Hypertonic saline enhances host response to bacterial challenge by augmenting receptor-independent neutrophil intracellular superoxide formation. Ann Surg 2003;238(2):249-257

41 Chen Y, Bao Y, Zhang J, et al. Inhibition of neutrophils by hypertonic saline involves pannexin-1, CD39, CD73, and other ectonucleotidases. Shock 2015;44(3):221-227
42 Loomis WH, Namiki S, Hoyt DB, Junger WG. Hypertonicity rescues $\mathrm{T}$ cells from suppression by trauma-induced anti-inflammatory mediators. Am J Physiol Cell Physiol 2001;281(3): C840-C848

43 Attuwaybi B, Kozar RA, Gates KS, et al. Hypertonic saline prevents inflammation, injury, and impaired intestinal transit after gut ischemia/reperfusion by inducing heme oxygenase 1 enzyme. J Trauma 2004;56(4):749-758, discussion 758-759

44 Alam HB, Stanton K, Koustova E, Burris D, Rich N, Rhee P. Effect of different resuscitation strategies on neutrophil activation in a swine model of hemorrhagic shock. Resuscitation 2004;60(1):91-99

45 Parker LC, Whyte MKB, Dower SK, Sabroe I. The expression and roles of Toll-like receptors in the biology of the human neutrophil. J Leukoc Biol 2005;77(6):886-892

46 Lohman AW, Isakson BE, Differentiating connexin hemichannels and pannexin channels in cellular ATP release. FEBS Lett 2014;588(8):1379-1388

47 Madden JR, Dobyns E, Handler M, Foreman NK. Experience with electrolyte levels after craniotomy for pediatric brain tumors. J Pediatr Oncol Nurs 2010;27(1):21-23 